\title{
Outcome after Surgical Treatment of a Rare Congenital Pouch Colon Variant with Tubularized Coloplasty
}

\author{
Oliver Renz ${ }^{1}$, Paul Hechenleitner ${ }^{1}$, Beatrice Häussler ${ }^{1}$, Bettina Härter ${ }^{1}$ and Murat Sanal ${ }^{1,2^{*}}$ \\ ${ }^{1}$ Department of Pediatric Surgery, Medical University Innsbruck, Innrain 52, Christoph-Probst-Platz, 6020 Innsbruck, Austria \\ ${ }^{2}$ Department of Pediatric Surgery, Children's Hospital Bursa, Turkey
}

\section{Abstract}

Congenital pouch colon (CPC) is an unusual malformation characterized by dilatation of a shortened colon associated with an anorectal malformation (ARM). We report about three patients transferred to our department with the initial diagnosis of an imperforate anus. A rare form of complete congenital pouch colon (CCPC) without a genitourinary fistula was diagnosed during the preoperative diagnostic evaluations at our institute. Definitive surgery was performed by tube coloplasty of the colonic pouch segment and posterior sagittal anorectoplasty (PSARP). Long term follow-up at a range of one to three years was fairly satisfactory in terms of function and without any loco-regional complications.

\section{Publication History:}

Received: July 05, 2016

Accepted: September 08, 2016

Published: September 10, 2016

\section{Keywords:}

Congenital pouch colon, Anorectal Malformation, Tubularized coloplasty

\section{Introduction}

$\mathrm{CPC}$ is an unusual malformation consisting of a shortened colon combined with a pouch like dilatation associated with anorectal agenesis usually associated with a genitourinary fistula.

This condition has been classified by Narasimharao et al. [1] into 4 groups according to anatomical subtypes based on the length of colonic pouch in relation to normal colon. Subsequently extended ways of classification were published for example defining pouch colon in complete congenital pouch colon (CCPC) and incomplete congenital pouch colon (ICPC) [2]. The "Saxena-Mathur classification" categorizes CPC into 5 types regarding the anatomical morphology [3].

Due to variability of anatomical features and different clinical manifestations, there is a wide range of surgical treatment options of the CPC. Despite the possibility of using staged procedures with protective placement of stoma or using one stage procedure, two different main surgical strategies are discussed in literature. The first one is pouch resection and pull through procedure. The second strategy is tubularisation of the colonic pouch and pull through of the coloplasty [4-11].

We describe our surgical strategy in three cases and outcome of these patients.

\section{Patients and Methods}

All patients had a rare variant of CPC malformation and closely resembled each other with ileum opening directly into the colonic pouch (CCPC), without a genitourinary fistula.

They have been transferred to our institute after birth with the initial diagnosis of anorectal malformation with imperforate anus in a time period of ten years (2001-2011). None of them had prenatal screening tests or ultrasound examinations during pregnancy. Clinical examination revealed distended abdomen and perineal inspection showed no cutaneous fistula. Meconium was not detected in the urine (Table 1. Patient's characteristic). Based on further evaluations with ultrasound investigation and abdominal plain $\mathrm{x}$-ray CPC anomaly was suspected.

Transverse laparotomy was performed and exploration confirmed $\mathrm{CPC}$ in all patients. Because ileum opened directly into these colonic pouches we decided to perform a diverting ileostomy to save the colonic pouch for the second stage procedure. Approximately $15 \mathrm{~cm}$ proximal of the colonic pouch, a diverting stoma was created. With intra-operative contrast medium imaging of distal intestinal segment, we could verify that colonic pouches indeed had no connection with bladder or urinary tract (Figure 1).

There was no further associated gastrointestinal anomaly.

The distal tube of the ileostomy was irrigated twice a week with normal saline solution. After at least 6 months all three patients thrived well and no complication occurred during this period.

As second stage operation stapler tubularized coloplasty was performed (Figure 2).

After closure of the laparotomy incision, reconstruction of intestinal continuity was carried out by abdominal posterior sagittal "anocoloplasty" comparable to PSARP (posterior sagittal ano-rectoplasty) (Figure 3).

"Corresponding Author: Dr. Murat Sanal, Medical University Innsbruck, Department of Pediatric Surgery, Anichstr. 35, 6020 Innsbruck, Austria, Tel: + 43 51250480774 ; E-mail: murat.sanal@uki.at

Citation: Renz O, Hechenleitner P, Häussler B, Härter B, Sanal M (2016) Outcome after Surgical Treatment of a Rare Congenital Pouch Colon Variant with Tubularized Coloplasty. Int J Surg Surgical Porced 1: 109. doi: http://dx.doi. org/10.15344/ijssp/2016/109

Copyright: ( 2016 Renz et al. This is an open-access article distributed under the terms of the Creative Commons Attribution License, which permits unrestricted use, distribution, and reproduction in any medium, provided the original author and source are credited. 
Citation: Renz O, Hechenleitner P, Häussler B, Härter B, Sanal M (2016) Outcome after Surgical Treatment of a Rare Congenital Pouch Colon Variant with Tubularized Coloplasty. Int J Surg Surgical Porced 1: 109. doi: http://dx.doi.org/10.15344/ijssp/2016/109

Page 2 of 3

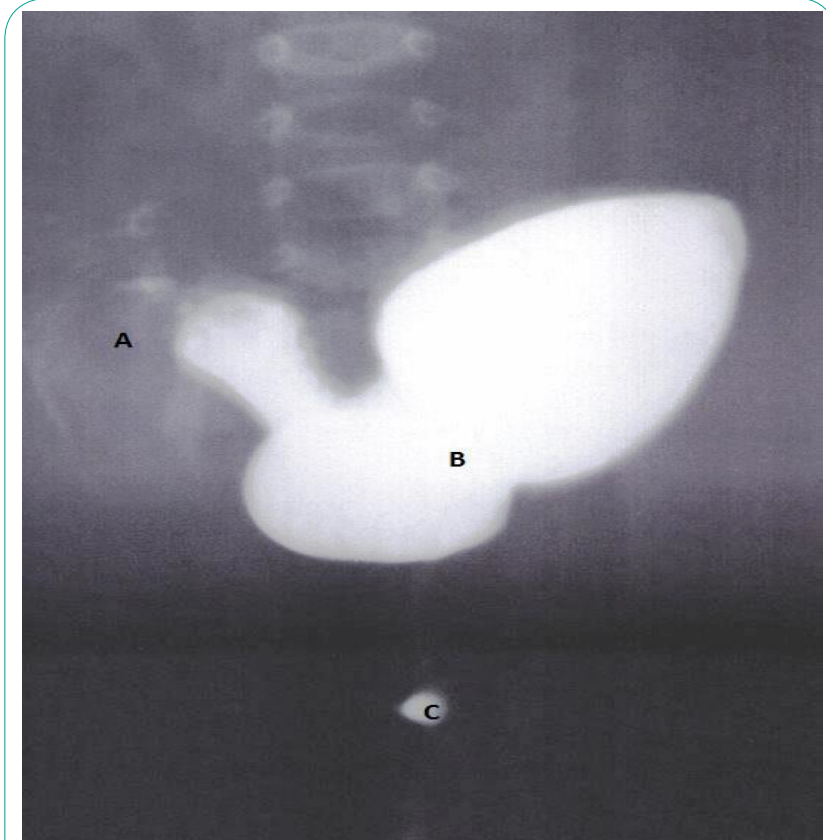

Figure 1: A terminal ileum filled with contrast, B contrast study depicting the huge dilated pouch colon, $\mathrm{C}$ contrast medium drop in the region of anal dimple, $\mathrm{B} / \mathrm{C}$ distance demonstrated between pouch colon and deposit of contrast medium localized at the area of anal dimple.

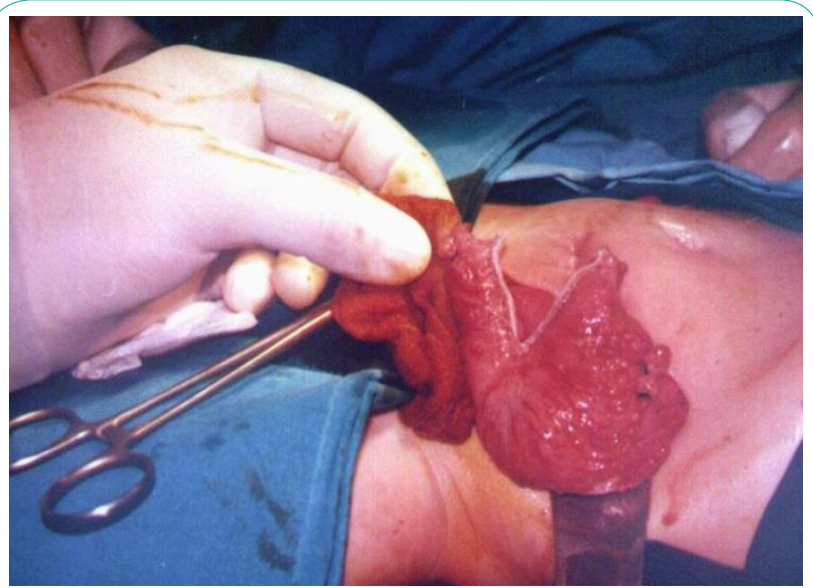

Figure 2: We created a tube of approximately $1.5-2 \mathrm{~cm}$ in diameter. A length of 10 to $12 \mathrm{~cm}$ was required to bring the tubularized colon tension free out as a "neoanus". The colonic tube was made by stapler resection of the colonic pouch along the whole circumference preserving the mesenteric vascular pedicle to ensure sufficient blood supply.

Postoperative wound care of the "neoanus" was performed with dressing material. Starting from the second postoperative week we performed anal dilatation protocol according to Pena guidelines [12] continuing for at least six months.

Subsequently, after complication-free healing process the diverting ileostomy was closed with end-to- end single layer anastomosis.

\section{Results}

None of the children had perioperative surgical complications. Only one patient suffered from excoriations circumferential in the area of the "neoanus", which could be healed by local treatment with zinc ointment.

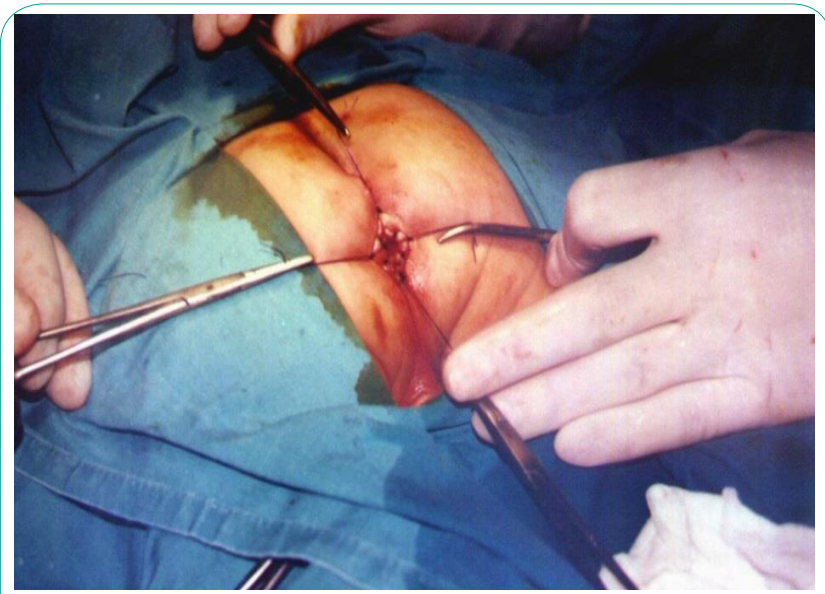

Figure 3: Bleeding at the mucosal end of the tube should be sufficient to confirm properly healing of "ano-coloplasty" sutures.

Initially the frequency of bowel movement was 8 to 7 per day, but it decreased to just four to two times daily. After three months all patients had semi formed stools. Two patients had postoperative clinical follow-up for three years and the third patient for only two years. None of the patients developed long term complications, neither pouch re-dilatation nor anoplasty stricture.

During the entire follow-up period these children thrived well and had permanently semi formed stool two to four times per day.

\section{Discussion}

Congenital pouch colon is a rare variant of anorectal malformation described in the literature with $9 \%$ [7] and $6.3 \%-15.1 \%$ [9]. Our three cases represent a very rare form of CPC type I [1] without genitourethral fistula. In the literature Mathur et al. described one case out of 80 [4], Chadha et al. 3 cases out of 41 [7] and Sharma et al. 3 out of 68 cases [11].

Large series of the CPC anomalies were reported mostly from India and neighboring nations in Asia pointing out the high complication potential of surgical management and functional outcome.

Many of these reports addressed possible reasons for severe complications like re-dilatation of the tubularized colonic pouch after pull-through surgery $[4,6,8,10,11]$. Gangopadhyaya et al. reported in their large series on the histopathologic examinations of the resected pouches and pointed out that these structures have abnormally developed tissue and need to be resected for better functional outcome [9]. Other authors focused on the surgical technique performing tubular colorraphy $[5,8,10]$. With our technique of creating coloplasty tube we reiterate these recommendations concerning size and length of colonic tube and fortunately we did not see this re-dilatation phenomenon during our observation period.

Stricture of the anoplasty after PSARP is a well-known complication and this kind of stricture might influence the formation of a re-dilatation of the tubularized colon. Therefore in our opinion long-term dilatation of the "neoanus" is a very important fact. We provided close outpatient follow-up of our patients keeping strictly to the dilatation protocol according to Pena [12] for at least 6 months. Overall we assume some selective advantage for PSARP surgical procedure due to a lack of genitourinary fistula in our selected small patient collective. 
Citation: Renz O, Hechenleitner P, Häussler B, Härter B, Sanal M (2016) Outcome after Surgical Treatment of a Rare Congenital Pouch Colon Variant with Tubularized Coloplasty. Int J Surg Surgical Porced 1: 109. doi: http://dx.doi.org/10.15344/ijssp/2016/109

Page 3 of 3

Main functional postoperative problems of the children with CPC are high frequency bowel movements with aqueous stools, high stool frequency and perineal excoriations. All these gastrointestinal complications may cause a failure to thrive and a loss of quality of life. Poor continence with high stool frequency was described after pullthrough of ileum in patients with CPC type I/II [6]. Our intention to retain a tubularized pouch colon was to reach continence to a certain extend in contrast to ileum pull through. Further we intended to avoid constructing a technical complex J-shaped or S-shaped ileum reservoir and wanted to prevent a permanent abdominal enterostomy.

However despite the fact of already published large series in literature the decision for choosing the adequate surgery method and subsequently achieving a good quality of life for these patients is not easy.

In conclusion concerning the very rare variant of CCPC without genitourinary fistula, we can speculate that coloplasty with posterior sagittal "ano-coloplasty" followed by anal dilatations works well, and that these patients may have a fairly good outcome. Nevertheless it should be reevaluated in the long term.

\section{Competing Interests}

The authors declare that they have no competing interests.

\section{References}

1. Rao KLN, Yadav K, Mitra SK (1984) Congenital short colon with imperforate anus (pouch colon syndrome). Ann Pediatr Surg 1:159-167

2. Gupta DK, Sharma S (2006) Congenital pouch colon. In: Anorecta Malformations, 1st ed. Hutson J, Holschneider A, editors. Springer Heidelberg, Chapter 11: p. 211-222.

3. Saxena AK, Mathur P (2008) Classification of congenital pouch colon based on anatomic morphology. Int.J. Colorectal Dis 23: 635-639.

4. Mathur P, Saxena AK, Simlot A (2009) Management of congenital pouch colon based on the Saxena-Mathur classification. J Pediatr Surg 44: 962966.

5. Gupta DK (2007) Congenital pouch colon: Present lacunae. J Indian Assoc Pediatr Surg 12: 1-2.

6. Puri A, Chadha R, Choudhury SR, Garg A (2006) Congenital pouch colon follow-up and functional results after definitive surgery. J Pediatr Surg 41:1413-1419

7. Chadha R, Bagga D, Malhotra CJ, Mohta A, Dhar A, et al. (1994) The embryology and management of congenital pouch colon associated with anorectal agenesis. J Pediatr Surg 29: 439-446.

8. Chadha R, Bagga D, Gupta S, Prasad A (2002) Congenital Pouch Colon Massive Redilatation of the Tubularized Colonic Pouch After Pull-Through Surgery. J Pediatr Surg 37: 1376-1379.

9. Gangopadhyay AN, Patne SC, Pandey A, Aryya NC, Upadhyaya VD, et.al. (2009) Congenital pouch colon associated with anorectal malformationhistopathologic evaluation. J Pediatr Surg 44: 600-606.

10. Wakhlu A, Wakhlu AK (2009) Technique and long-term results of coloplasty for congenital short colon. Pediatr Surg Int 25: 47-52.

11. Sharma S, Gupta DK (2015) Management options of congenital pouch colon-a rare variant of anorectal malformation. Pediatr Surg Int 31: 753758.

12. Peña A (1990) Atlas of surgical management of anorectal malformations Postop care, complications and results. Springer, New York, USA, Chap 7: $92 \mathrm{p}$. 